\title{
Object-Oriented Modelling and Simulation of a Molten-Salt Once-Through Steam Generator for Solar Applications using Open-Source Tools
}

\author{
Francesco Casella Stefano Trabucchi \\ Dipartimento di Elettronica, Informazione e Bioingegneria, Politecnico di Milano, Italy, \\ \{francesco.casella, stefano.trabucchi\}@polimi.it
}

\begin{abstract}
Concentrated solar power plants (CSP) coupled with thermal storage have the potential of being competitive with conventional fossil fuel and hydro power plants, in terms of dispatchability and provision of ancillary services. To achieve this potential, the plant design has to be focused on flexible operation, which is the main goal of the PreFlexMS Horizon 2020 European research project. This can be achieved by the integration of a Molten Salt Once Through Steam Generator within the power unit, an innovative technology with greater flexibility potential if compared to steam drum boilers, currently the state of the art in CSP. Given the focus on flexible operation, dynamic modelling and simulation from the early design stages is of paramount importance, to assess the plant dynamic behaviour and controllability, and to predict the achievable closed-loop dynamic performance, potentially saving money and time during the detailed design, construction and commissioning phases. The present paper aims to demonstrate that it is possible to achieve this goal by means of Modelica-based open-source libraries and tools.
\end{abstract}

Keywords: simulation, Modelica, solar power plants, once-through boilers, open-source tools.

\section{Introduction}

During the 2015 United Nations Climate Change Conference 195 countries agreed to reduce their green-house gases production "as soon as possible", in order to keep the global warming below $2{ }^{\circ} \mathrm{C}$. Research on Concentrated Solar Power (CSP) plants is therefore of paramount importance, since such plants could be able to fill the gap between renewable and fossil energy sources, in terms of predictability, flexibility and power production planning.

In this context, the PreFlexMS Horizon 2020 research project aims to design a $100 \mathrm{MW}_{\mathrm{el}} \mathrm{CSP}$ power unit with flexibility features comparable to state-of-the-art gas-fired combined cycle plants, and to demonstrate it on a scaleddown pilot plant. The two pillars of this project are: the optimized operation of the plant based on customized weather prediction and on the demand on the electrical market, on one side, and a power unit based on an innovative once-through molten-salt steam generation unit on the other side. The latter is expected to provide much more flexible operation than the current state of the art in CSP, i.e., steam-drum type steam generators.

Dynamic modelling and simulation is a key aspect of the project. The plant dynamic behaviour and controllability need to be assessed from the early design stages, and it is essential to demonstrate that the achievable closedloop performance of the plant is consistent with the project goals, which ask for: a) an overall hot start-up time of 30 minutes and b) ramping rates comparable to state-of-the-art combined-cycle plants.

The goal of this paper is to describe the modelling approach that was taken in this project, and in particular to demonstrate that object-oriented open standards and open source tools are perfectly suited to carry out this task.

The paper is structured as follows. Section 2 introduces the modelling methodology, language, model libraries and simulation tools that were used in the project. The plant subject of the study is described in Section 3; as to its modelling, Section 4 discusses new device models that were created for this project, while Section 5 discusses the overall plant model. The simulation objectives and methodology is presented in Section 6, while simulation results are presented in Section 7. Finally, Section 8 concludes the paper.

\section{Modelling methodology, libraries and tools}

The equation-based, object-oriented modelling approach (EOOM) and the Modelica language have been employed for this work. A general discussion of this topic goes beyond the scope of this paper, the interested reader is referred to (Mattsson et al., 1998; Tiller, 2001; Fritzson, 2014). We only stress two key points here: on one hand, that dynamic models are described by means of declarative descriptions, based on differential-algebraic equations, and of modular composition through a-causal physical connectors; on the other hand, that the Modelica language used to code the model is a non-proprietary, toolindependent standard.

A key feature of the EOOM is that it promotes reusability, while at the same time allowing for extensions and customizations with a minimal effort. This was also 
the case for the present study, which relied on existing open-source model libraries. Besides the Modelica Standard Library, which contains utility models as well as the strategically important IAPWS IF97 water/steam model, the ThermoPower and the IndustrialControlSystem Modelica libraries were used. The first one is a library for the modelling of thermal power plants, whose design principles are discussed in (Casella and Leva, 2006) and that has been used, adapted and validated extensively to model various types of power generation and energy conversion systems such as steam generators (Casella and Leva, 2005), combined-cycle power plants (Casella and Pretolani, 2006; Bartolini et al., 2012), nuclear power plants (Cammi et al., 2011), cryogenic systems (Zanino et al., 2013), and Organic Rankine Cycle systems (Casella et al., 2013). The second one is the IndustrialControlSystem library (Bonvini and Leva, 2012), containing detailed models of industrial PID controllers endowed with features such as anti-windup, output tracking, etc., that were needed for the modelling of the control system.

Device models not found in ThermoPower had to be adapted or written from scratch, see Section 4.

In principle, any Modelica-compliant simulation tool can be used to analyze and simulate it. In this paper, we discuss the results obtained by using the open-source OpenModelica tool, to demonstrate that the dynamic modelling and simulation task can be carried out using a completely open-source tool chain.

\section{Plant Description}

The subject of this study is an innovative $100 \mathrm{MW}_{\mathrm{el}}$ Concentrated Solar Power (CSP) plant with thermal storage. The solar field directs the solar radiation onto the receiver tower, where a mixture of Sodium and Potassium nitrate (commonly called hereafter molten salt (MS)) is heated up to $565^{\circ} \mathrm{C}$ and stored in a tank. The molten salt (MS) acts both as Heat Transfer Fluid (HTF) and as Thermal Energy Storage (TES). In the context of this study, whose scope is limited to the power generation block, the hot MS is considered as coming from a source point at constant temperature.

The model represents the power unit, composed of the steam generator system (SGS), the steam turbine, the feedwater heaters train with a deaerator, and the plant control system. The SGS is the core of the dynamic analysis, while the turbine and the feedwater heaters provide the correct boundary conditions for the boiler.

\subsection{Innovative aspects}

The main innovative aspect of the present plant is the introduction of a Molten Salt Once-Through Steam Generator (OTSG) instead of a drum-type boiler, which represents nowadays the state of the art concerning CSP with thermal storage. Such a system can provide a higher plant flexibility, since the drum and its related large inertia and thermo-mechanical stresses are absent.

The OTSG is a continuous flow path of Shell\&Tube heat exchangers, whose mechanical design is optimized in order to maximize the life-cycle of the whole boiler. The MS flow is cooled down to the design value of $290^{\circ} \mathrm{C}$ while on the other side superheated steam is produced.

Another non-conventional issue is represented by the hot fluid thermal inertia. Compared to once-through boilers used in advanced combined-cycle (CC) plants, where the thermal inertia of the hot fluid is negligible, the heat capacity of the MS in this plant is the dominant thermal storage in the process, so that the thermal coupling between the MS and the water/steam side and in general the plant dynamic response is quite different from that of existing CC plants.

\subsection{OTSG topology}

As the plant is meant to operate in sliding pressure mode, the joint effect of evaporation and bleed steam pressure reduction would significantly decrease the MS OTSG outlet temperature and the feedwater (FW) temperature at the same time, during part-load operation. This phenomenon is not acceptable, because it could lead to MS freezing, which is a catastrophic fault. Thus, the process is designed so that the MS outlet temperature is kept almost constant as the plant load is reduced.

The key aspects of the heat exchangers topology are the following (see Figure 1):

- the greater amount of feedwater mass flow enters the OTSG at the economizer (ECO) cold end, flows through the evaporator (divided into EVA1 and EVA2) and enters a phase separator. If the steam quality at the EVA2 hot end is equal to one, then the separator does not affect the steam thermodynamic condition; otherwise, the saturated steam enters the superheating section $(\mathrm{SH})$ and the saturated liquid is collected at the separator bottom and recirculated at the OTSG inlet;

- a small amount of feedwater mass flow enters a MS heater, and is then recirculated to the last high pressure feedwater heater cold end;

- from the MS line point of view, the steam SH and reheating $(\mathrm{RH})$ sections run in parallel: the hot MS mass flow entering the OTSG is split up in two branches by means of a valve, and then mixed at the evaporation section hot end;

- a by-pass valve on the last high pressure feedwater heater offers another degree of freedom, useful to obtain the desired feedwater temperature at OTSG inlet.

Contrary to the bottoming cycle of a modern combined cycle, feedwater heaters are needed in order to provide a sufficiently high water temperature at the OTSG inlet and to prevent the MS from freezing.

The result is a non-conventional steam Rankine cycle, with a high number of recirculation flows interacting in a non-trivial way, thus making the dynamic simulation a key factor during system and control design. 


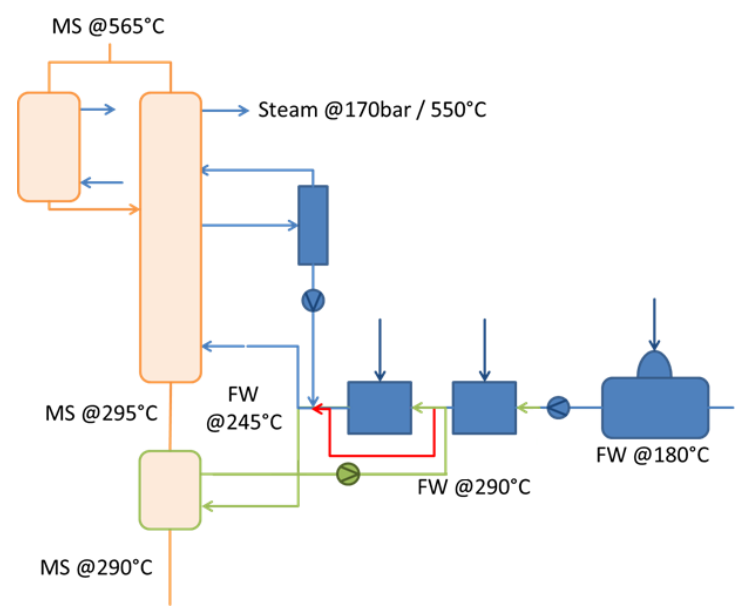

Figure 1. OTSG and high pressure feedwater train topology, patent pending EP 15290109.6-1610

\section{Device modelling}

As a first step, all the physical devices of the system are modelled, by means of both re-used and custom models.

\subsection{Re-used components}

The dynamic properties of a thermal power system are mainly given by the heat exchangers design, in terms of heat exchange surface, volumes and metal masses. These parameters are set within the FlowlDFV component of the ThermoPower library, which represents by means of 1D finite volumes method the hot and cold paths of each heat exchanger. The two equivalent tubes are connected through the model of a thin metal wall, where the heat exchanger mass is concentrated. Finally, the heatExchangeTopology component lets the user set the flow path displacement, such as co-current, counter-current and Shell\&Tube (Boni, 2013).

Other simple components have been taken directly from the ThermoPower library, such as liquid and steam valves, pressure drops, and pumps, and they are connected between each others through standard Modelica stream connectors (Franke et al., 2009). The water/steam thermodynamic properties are given by the IF97 tables of the Modelica standard library.

\subsection{Custom component models}

The complexity and non-standard features of the power unit required the development of custom models of specific components, in order to represent in the best way possible their physical behaviour.

\subsubsection{Separator}

At the end of the evaporation section, a two-phase cyclone separator guarantees that only saturated or superheated steam enters the superheating section, so that no liquid drops can reach the turbine inlet. Since from the geometrical point of view this device is a high and nar- row cylinder with a small cross sectional area and the inlet flange positioned in the upper part, it can be assumed that the thermal contact between the liquid possibly present in the lower part and the wet/dry steam is negligible.

The four main equations describing the dynamics of the separator are thus the mass and energy balances over the liquid and steam volumes, assuming as states the liquid level $l$, the liquid enthalpy $h_{l}$, the pressure $p$ and the vapour enthalpy $h_{v}$.

The outlet steam condition can be either saturated or superheated, depending on the steam quality at the evaporators outlet. The following equation guarantees that no wet steam enters the $\mathrm{SH}$ :

$$
h_{v, \text { out }}=\max \left(h_{v}, h_{v, \text { sat }}(p)\right)
$$

\subsubsection{Feedwater heater}

A feedwater heater is a particular heat exchanger where the steam extracted from the turbine condenses on a tubes bundle. The feedwater flow inside the bundle sets the amount of steam that actually condenses: the lower the feedwater mass flow, the lower the condensing mass flow.

The mechanical design of such an heat exchanger usually assumes three different heat exchange zones:

1. a desuperheating section, where the bleed steam from the turbine is brought to saturation condition. Since the bled steam is not always superheated, this zone could be missing, especially in low pressure heaters train;

2. a condensing section, where the bleed steam and the drained subcooled liquid coming from the higher pressure heaters mix up and condense;

3. a subcooling section, where the condensate is subcooled.

Such a structure has been modelled as three heat exchangers in series, once more making use of the component FlowlDFV of the ThermoPower library, as shown in Figure 2. A valve downstream the steam subcooling section controls the condensate level inside the condensing section by means of a PI controller, discharging the drained flow into the next, lower pressure heater.

The key base component of the feedwater model is the steam condenser, called NusseltCondenser. It is modelled as a cavity where the steam fraction coming from the drained and the bleed flows condenses. As for the separator, the four main equations describing the dynamics of the separator are the liquid and steam mass and energy balances, assuming as states the liquid level $l$, the liquid enthalpy $h_{l}$, the pressure $p$ and the vapour enthalpy $h_{v}$.

Thanks to the min and max operators and assuming that the liquid entering the cavity goes directly into the liquid pool at the shell bottom, the energy balances can cover also the cases where the entering steam is wet, usually at part load. 


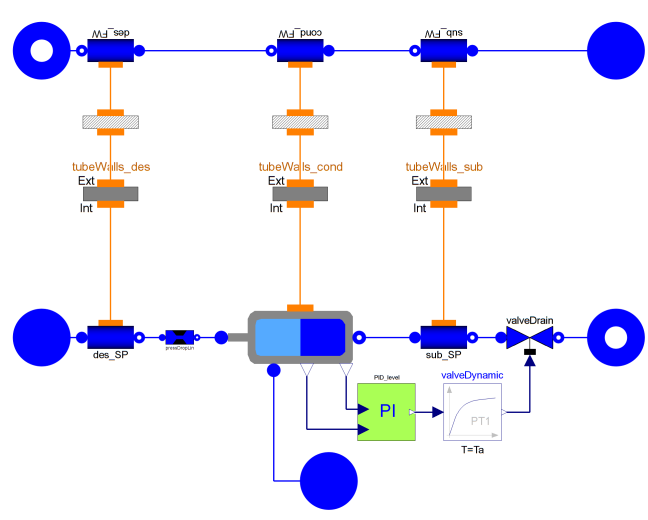

Figure 2. Object diagram of a feedwater heater

The tube bundle on which steam condensation takes place is divided into $N_{w}$ finite volumes, as the Distribuited Heat Transfer (DHT) connectors in Figure 2 shows. This means that the total condensate mass flow and heat flux is the sum of the single contributions of each volume. The equations for the $i-t h$ volume read:

$$
\begin{aligned}
& Q_{i}=\max \left[0, \frac{\gamma S}{N_{w}}\left(T_{s a t}-T_{c, i}\right)+w_{c, i}\left(h_{v}-h_{v, s a t}\right)\right] \\
& w_{c, i}=\frac{Q_{i}}{h_{v}-h_{c, i}} \\
& \text { condState }_{i}=\text { setState } \_p T\left(p, T_{c, i}\right) \\
& h_{c, i}=\min \left[{\text { specificEnthalpy } \left.\left(\text { condState }_{i}\right), h_{l, s a t}\right]}^{2}\right. \\
& T_{c, i}=\frac{T_{w, i}-T_{\text {sat }}}{2}
\end{aligned}
$$

where the subscript "c" refers to the condensate condition and "w" to the metal tubes. Thus:

$$
\begin{aligned}
& Q_{\text {flux }}=\sum_{i=1}^{N_{w}} w_{c, i} h_{c, i} \\
& Q_{\text {cond }}=\sum_{i=1}^{N_{w}} Q_{i} \\
& w_{\text {cond }}=\sum_{i=1}^{N_{w}} w_{i}
\end{aligned}
$$

where $Q_{\text {cond }}$ refers to the heat power entering the metal wall, $w_{\text {cond }}$ the actual condensate flow and $Q_{\text {flux }}$ the global enthalpic flux entering the liquid pool through by means of the condensate.

During some transients the liquid enthalpy could become greater than the saturated liquid enthalpy, so that a little amount of liquid evaporates. Hence, the following term has been added within the mass balances:

$$
w_{e v}=\frac{x_{l} \rho_{l} V_{l}}{\tau_{e v}}
$$

where $x_{l}$ is the steam fraction in the liquid volume and $\tau_{e v}$ is an equivalent rising time, a tuning parameter.

\subsubsection{Deaerator}

The deaerator is a component of paramount importance within a steam cycle. Its main function is to extract from the feedwater flow the incondensable gases, which would chemically react at high temperature compromising the lifetime of high-temperature components such as the $\mathrm{SH}$ and the high pressure turbine.

It is worth noticing that the presence of incondensable gases and the steam blown away through the discharge valve can be omitted entirely from the model, due to their very modest quantities, while the hydraulic and thermal effects the deaerator has on the rest of the cycle must be taken into account, due to its large size.

The most efficient working condition for such a component is the thermodynamic equilibrium between steam and water, since it promotes the gases dissolution from feedwater mass flow. Under the assumption that the design and the local control system of this device guarantee the optimal condition, it can be modelled as a parallelepipedshaped vessel at thermodynamic equilibrium, where all the entering flows mix up.

Assuming as states the pressure $p$ and the mixture enthalpy $h$, the main model equations are the mass and energy balances on the device. The liquid level can be derived from the steam quality, as follows:

$$
l=H\left(1-\frac{\rho}{\rho_{v, \text { sat }}} x\right)=H\left(1-\frac{\rho}{\rho_{v, \text { sat }}} \frac{h-h_{l, s a t}}{h_{v, \text { sat }}-h_{l, \text { sat }}}\right)
$$

\subsubsection{MS fluid model}

The MS has been represented as an extension of the Modelica.Media.Interfaces.PartialPureSubstance class. References value for enthalpy, density and temperature have been set according to the specific MS thermo physical properties. Density and specific heat capacity are considered as linear functions of the temperature.

\section{Plant modelling}

The complete plant model is composed by the four macro components described in Sec. 3. Thanks to the encapsulation feature of the Modelica language, each component can be easily replaced with more or less detailed ones, without modifying the rest of the system. Since there are several possible plant operational modes that have been tested, this aspect hugely reduced the time spent for test cases setup.

\subsection{OTSG model}

The OTSG model follows the process flow diagram of Figure 1. Six heat exchanger models are connected via stream connectors, within a base class model that contains the common OTSG fluid and control signal interfaces.

Two important aspects concerning the pressure drops representation have to be highlighted:

- the MS pressure drops are neglected. As the MS is always in liquid state, its density and specific heat capacity are hardly influenced by the fluid pressure; 


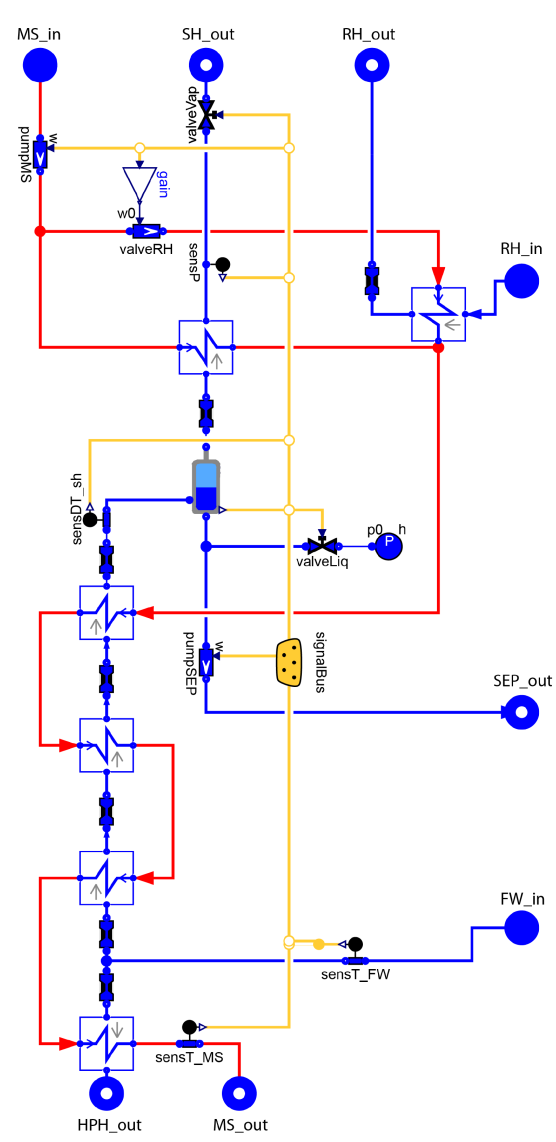

Figure 3. Object diagram of the OTSG

- the steam/water pressure drops are taken into account through the ThermoPower component $\mathrm{Wa}$ ter.PressDrop at the inlet or the outlet of each heat exchanger. This approach have two main advantages: first, the coupling between mass and energy balances is reduced, so that the resulting algebraic loops are easier to be solved; secondly, each heat exchanger is independent from the equations system point of view, so that the resulting symbolic manipulation produces a more efficient simulation code.

The blue lines in the figure refer to the water/steam flows, the red lines to the MS flows and the yellow lines to the control signals. The water/steam pressure drop components are the blue rectangular boxes in between the heat exchangers, while the gain block splits the MS mass flow as required between $\mathrm{SH}$ and RH branches. Heat exchangers and flow lines parameters have been taken from design Process Flow Diagrams (PFD) and heat exchangers datasheets.

\subsection{Turbine model}

The turbine model was built by connecting several ThermoPower components Water.SteamTurbineStodola, each representing a turbine section with many stages, and accounting for feedwater heater steam extractions and for leakage and sealing flows. The model parameters (Stodola's law flow coefficient and isentropic efficiency) have been extrapolated from the PFDs. Finally, all the turbine sections are connected through mechanical interfaces representing the common turbine shaft.

\subsection{Feedwater preheaters train}

The power plant layout features a high pressure and a low pressure feedwater heater train, with the deaerator in between. The feedwater models have been connected to reproduce the topology reported in Sec. 3.

\subsection{Plant control system}

Once the single devices have been tuned and tested, the overall open-loop system dynamics has been thoroughly analysed, in order to develop a control architecture and controller tunings that were able to meet the control requirements.

More specifically, the model was numerically linearized at different operating load levels. The linearized models allowed to compute step responses, input-output transfer functions and frequency responses (Bode diagrams), as well as the Relative Gain Array (RGA) matrix, which allowed to investigate the couplings between the different control loops. This analysis led to the definition of a decentralized control strategy based on PID controllers and static feed-forward set-point compensation. Figure 4 shows the block diagram of one of these controllers, which are also defined by Modelica code.

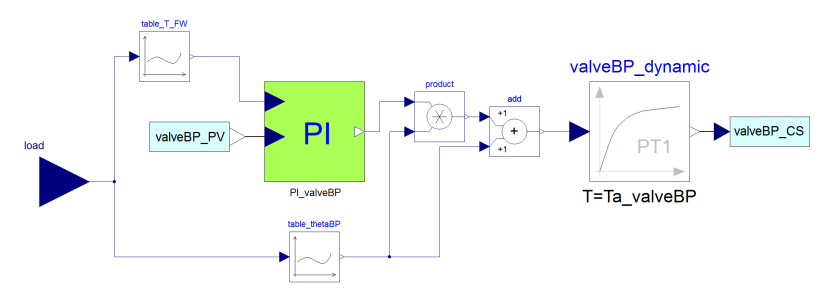

Figure 4. Object diagram of the high-pressure feedwater heaters by-pass control loop

\subsection{Complete plant model}

All the macro components can be aggregated in a single global system model, representing the whole power unit of the CSP plant. In Figure 5 the complexity of the system is evident, thus only the high-pressure part of the plant has been considered. This simplification does not affect the analysis results: the big size of the deaerator, in fact, dynamically decouples the high pressure from the low pressure part of the plant.

\section{Simulation objectives - methodology}

The simulation campaign aims to demonstrate that such a complex system can be simulated with open-source tools (e.g. OpenModelica). In particular, three different test cases have been analysed:

- A - nominal load: simulation of the nominal plant regime, to check the static correctness of the model; 
TABLE 1. Machine Features

\begin{tabular}{ccc}
\hline & Laptop & Workstation \\
\hline CPU & Intel(R) Core(TM) i7-4810MQ & Intel(R) Xeon(R) E5-2650 v3 \\
Clock & $2.8 \mathrm{GHz}$ & $2.3 \mathrm{GHz}$ \\
RAM & $8 \mathrm{~GB}$ & $72 \mathrm{~GB}$ \\
\hline
\end{tabular}

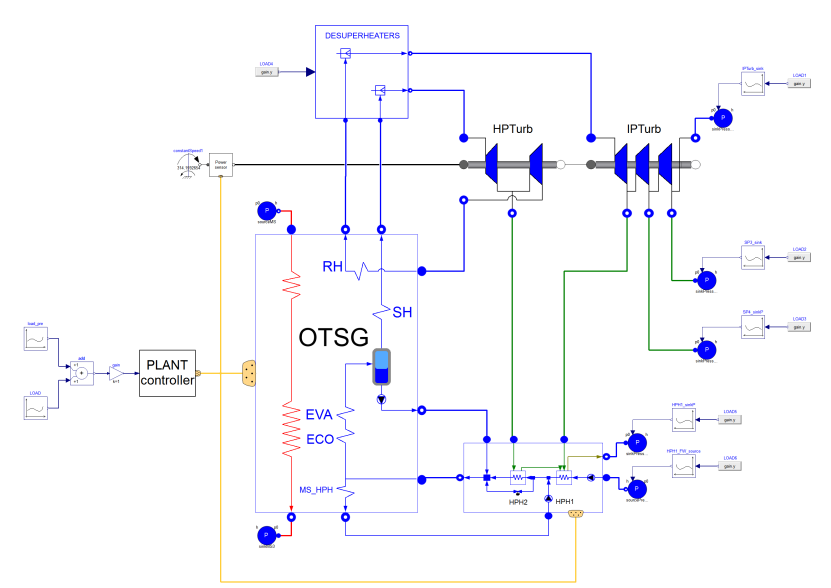

Figure 5. Object diagram of the high-pressure part of the power unit, used for daily start-up simulation

- B - load ramps: simulation of a series of load ramps to check the precision and robustness of the developed control system. The model is initialized at $100 \%$ load, brought to $60 \%$ with a slow negative ramp and then back to $100 \%$ by ramps having a $10 \%$ amplitude and a 10\%/min slope;

- $C$ - plant hot start-up: simulation of the daily power plant hot start-up. For numerical reasons, the model is first initialized at $40 \%$ load and brought to the initial load level of $20 \%$ with a slow negative ramp. Then, during the actual start-up simulation the load is brought back to $100 \%$ in 9 minutes.

The models have been compiled and simulated on a Linux workstation and on a Windows laptop, whose features are reported in Table 1. The sizes of the three models are summarized in Table 2.

\section{Simulation performance}

The thorough description of the simulated transients goes beyond the scope of this paper. For the sake of the exam-

TABLE 2. Test case models information

\begin{tabular}{ccc}
\hline Case & $\begin{array}{c}\text { no. } \\
\text { States }\end{array}$ & $\begin{array}{c}\text { no. } \\
\text { Variables }\end{array}$ \\
\hline A & 254 & 6945 \\
B & 254 & 6982 \\
C & 301 & 7554 \\
\hline
\end{tabular}

ple, we report the total mechanical power output and the temperatures of the feedwater and molten salt at the OTSG cold end, which should never go below $245^{\circ} \mathrm{C}$ during the hot start-up transient. The power set-point is closely followed by the actual value, with a small glitch when the operating mode of the evaporator is switched from wet operation with recirculation to dry, once-through mode. The MS temperature is always above the safety lower limit and the feedwater temperature closely follows its set-point (Figure 6).
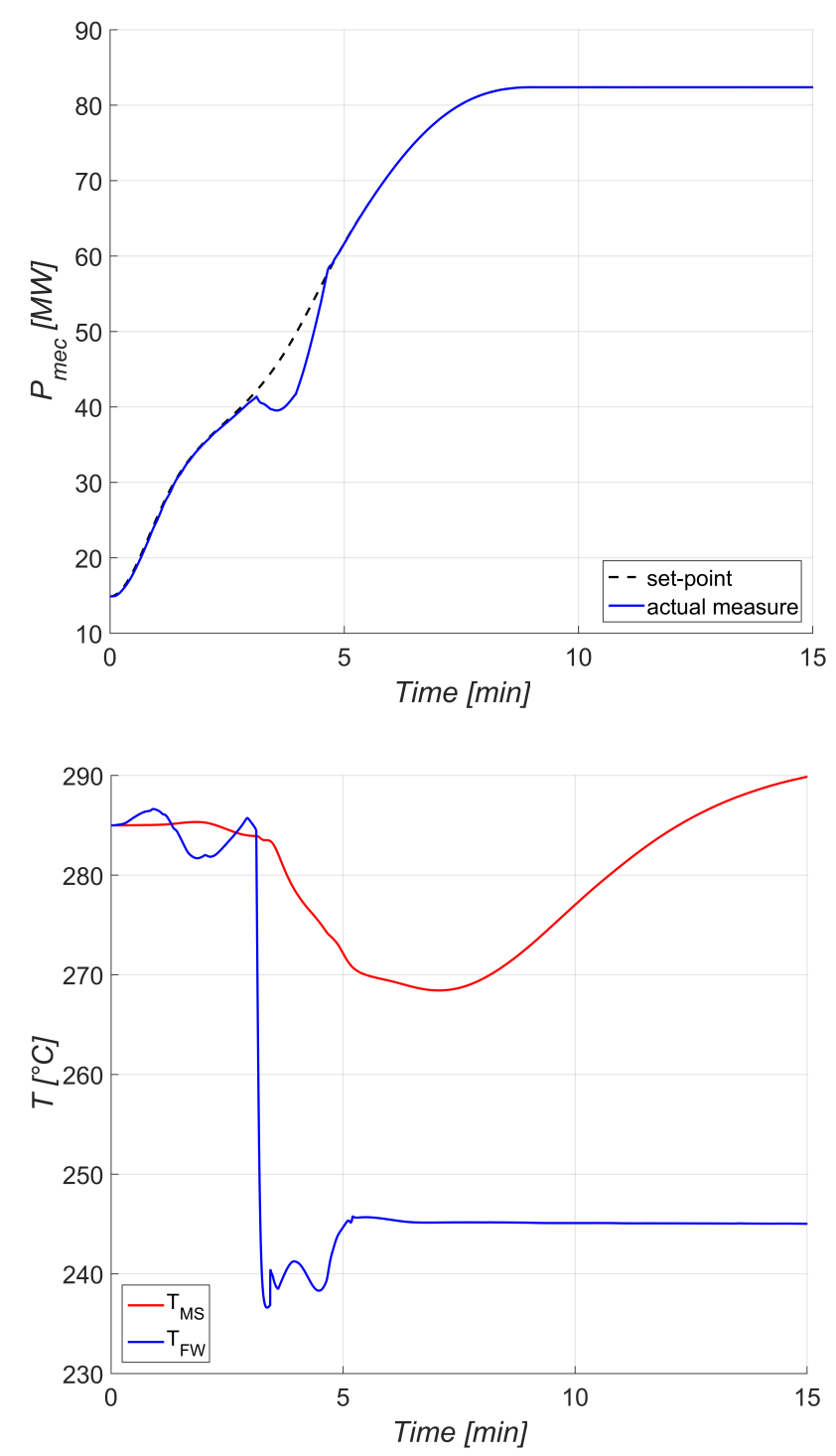

Figure 6. Mechanical power, MS and feedwater temperatures during hot start-up 
TABLE 3. Simulation performance

\begin{tabular}{ccccccc}
\hline Case & \multicolumn{2}{c}{$\begin{array}{c}\text { CPU compilation } \\
\text { time }[\mathrm{s}]\end{array}$} & \multicolumn{2}{c}{$\begin{array}{c}\text { no. integration } \\
\text { steps }\end{array}$} & \multicolumn{2}{c}{$\begin{array}{c}\text { CPU integration } \\
\text { time }[\mathrm{s}]\end{array}$} \\
& Linux & Windows & Linux & Windows & Linux & Windows \\
\hline A & 5.7 & 32 & 2898 & 3062 & 161 & 170 \\
B & 5.5 & 19 & 11030 & 10768 & 670 & 636 \\
C & 6.7 & 22 & 6404 & 6635 & 410 & 390 \\
\hline
\end{tabular}

The test campaign confirms the capability of OpenModelica to compile and simulate correctly complex models with a high number of states and variables. Table 3 reports the simulation performance for all the three test cases considered. The numerical algorithm employed is DASSL, without equidistant grid result interpolation, and with a relative tolerance of $10^{-6}$.

\section{Conclusions}

The results presented in this paper demonstrate that it is possible to tackle the detailed, system-level dynamic modelling and simulation of innovative power generation system for use in solar thermal power plants, using exclusively open modelling languages and simulation tools.

Modelica was chosen as the modelling language, since it is a tool-independent language that can also be used with open-source simulation tools, and for which many opensource modelling libraries are currently available.

The plant model was developed by extensively re-using basic models from the open-source ThermoPower library, with some adaptations and extensions. This model was first used to carry out extensive open-loop dynamic analysis, which allowed to design appropriate control strategies. The control system model was then also implemented in Modelica, using the IndustrialControlSystems open-source library, allowing to validate the closed-loop performance in non-trivial scenarios such as the hot startup of the plant.

Both open-loop and closed-loop transients have been simulated by means of the open-source OpenModelica tool. The simulation performance is satisfactory: the simulation code is compiled from the Modelica source code in a few seconds, and the most demanding closed-loop transient is simulated in about ten minutes. Most of the simulation time (about $75 \%$ ) is actually spent computing the water-steam properties, since the accurate but computationally demanding IAPWS IF97 model is used for that purpose. Given the on-going developments of the OpenModelica tool, it is expected that much better performance could be achieved in the near future.

Last, but not least, the choice of open-source tools also allowed to freely share the model among the different partners of the PreFlexMS Horizon 2020 project, both in source-code form and as compiled executables, without any constraints due to software licensing issues.

\section{Acknowledgment}

This work has been supported by the European Commission under Grant Agreement 654984-PreFlexMS, within the Horizon 2020 research programme.

\section{References}

Andrea Bartolini, Francesco Casella, Alberto Leva, and Luca Savoldelli. Object-oriented simulation for primary reserve scheduling in a combined cycle power plant. In Proceedings of the 2012 IEEE Multi-conference on Systems and Control, pages 764-769, Dubrovnik, Croatia, 3-5 Oct 2012. IEEE. ISBN 978-1-4673-4505-7.

Stefano Boni. Sviluppo di una libreria di componenti riconfigurabili per la simulazione di impianti ORC. Master's thesis, Politecnico di Milano, 2013.

Marco Bonvini and Alberto Leva. A Modelica library for Industrial Control Systems. In Proceedings 9th International Modelica Conference, pages 477-484, $\mathrm{Mu}-$ nich, Germany, Sep. 3-5 2012. The Modelica Association. doi:10.3384/ecp12076477.

Antonio Cammi, Francesco Casella, Marco Enrico Ricotti, and Francesco Schiavo. An object-oriented approach to simulation of IRIS dynamic response. Progress in Nuclear Energy, 53(1):48-58, Jan. 2011. doi:10.1016/j.pnucene.2010.09.004.

Francesco Casella and Alberto Leva. Object-oriented modelling $\&$ simulation of power plants with Modelica. In Proceedings 44th IEEE Conference on Decision and Control and European Control Conference 2005, pages 7597-7602, Seville, Spain, Dec. 12-15 2005. IEEE, EUCA. ISBN 0-7803-95689.

Francesco Casella and Alberto Leva. Modelling of thermohydraulic power generation processes using Modelica. Mathematical and Computer Modeling of Dynamical Systems, 12 (1):19-33, Feb. 2006. doi:10.1080/13873950500071082.

Francesco Casella and Francesco Pretolani. Fast start-up of a combined-cycle power plant: a simulation study with Modelica. In Christian Kral, editor, Proceedings 5th International Modelica Conference, pages 3-10, Vienna, Austria, Sep. 6-8 2006. Modelica Association. URL http: //www.modelica.org/events/modelica2006/ Proceedings/sessions/Sessionlal.pdf.

Francesco Casella, Tiemo Mathijssen, Piero Colonna, and Jos van Buijtenen. Dynamic modeling of organic rankine cycle power systems. Journal of Engineering for Gas Turbines and Power, 135(4):042310-1-12, 2013. doi:10.1115/1.4023120. 
R. Franke, F. Casella, M. Otter, M. Sielemann, H. Elmqvist, S. E. Mattsson, and H. Olsson. Stream connectors an extension of Modelica for device-oriented modeling of convective transport phenomena. In Proceedings 7th International Modelica Conference, pages 108-121, Como, Italy, Sep. 20-22 2009. The Modelica Association. ISBN 978-91-7393-513-5. doi:10.3384/ecp09430078. URL http://www.modelica.org/events / modelica2009/Proceedings/memorystick/ pages/papers/0078/0078.pdf.

P. Fritzson. Principles of Object Oriented Modeling and Simulation with Modelica 3.3. Wiley IEEE Press, 2014.
S. E. Mattsson, H. Elmqvist, and M. Otter. Physical system modeling with Modelica. Control Engineering Practice, 6 (4):501-510, 1998.

M. Tiller. Introduction to physical modelling with Modelica. Kluwer, 2001.

Roberto Zanino, Roberto Bonifetto, Francesco Casella, and Laura Savoldi Richard. Validation of the 4C code against data from the HELIOS loop at CEA Grenoble. Cryogenics, 53:25-30, 2013. doi:10.1016/j.cryogenics.2012.04.010. 